\title{
Comparaison des procédures de décentralisation et de négociation de la gestion de l'eau en France et en Californie
}

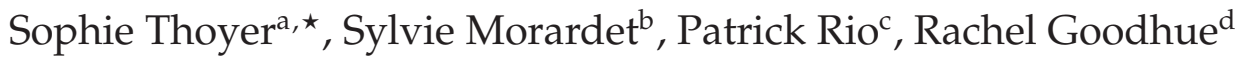 \\ a Économiste, ENSAM, département d'Économie, de Sciences sociales et de Gestion, UMR Lameta, 2 place Pierre Viala, \\ 34060 Montpellier cedex 1, France \\ b Économiste, Cemagref, Unité de recherche Irrigation, UMR Lameta (depuis novembre 2003, International Water Management \\ Institute, Private Bag X813, Silverton 0127, South Africa) \\ c Économiste, INRA-ESR, UMR Lameta, 2 place Pierre Viala, 34060 Montpellier cedex 1, France \\ d Économiste, University of California, Department of Agricultural and Resource Economics, One Shields Avenue, Davis, \\ California 95616-8512, USA
}

\begin{abstract}
Comment gérer une ressource renouvelable comme l'eau lorsque l'intensité des prélèvements se rapproche des limites des flux disponibles ou même les dépasse? Comment répartir la rareté, jusqu'au risque de manquer, entre des usagers aux intérêts opposés et hétérogènes? Droits d'usages, réglementations, systèmes de marché, instances de négociation, équipements pour stocker les ressources, autant de solutions combinées au sein des systèmes de gestion de l'eau. La comparaison de deux d'entre eux illustre un point insuffisamment souligné, à savoir que la négociation est aussi un miroir des pouvoirs qui lui font appel. En cela, NSS poursuit sa réflexion sur les formes politiques de la gestion des ressources.
\end{abstract}

La Rédaction

\section{Mots-clés :}

gestion concertée; résolution de conflit; participation; gestion de l'eau; bassin versant ; négociation

Keywords:
negotiated
management;
negotiation;
conflict resolution;
participation;
devolution;
water management

Résumé - Les procédures de décentralisation et de délégation de la gestion de l'eau sont comparées dans les cas californien et français pour mettre en évidence l'influence du système juridique des droits d'eau et de la participation des autorités publiques sur l'organisation des négociations et leur efficacité. On constate que le processus de négociation en Californie cherche à établir des compromis dans des situations de forte concurrence entre usagers de l'eau, alors qu'en France, il s'apparente plus à une procédure de concertation et de délégation. En Californie, les autorités publiques agissent comme un point de menace pour inciter les participants à trouver une solution. En France, l'intervention publique est plus directe puisqu'elle se charge d'établir de façon précise les règles de la négociation et se réserve le rôle de décideur en dernier ressort.

\footnotetext{
Abstract - Comparison of water management decentralisation and negotiation processes in France and California. The objective of the paper is to highlight the main differences between the French and the Californian decentralisation and devolution procedures for water management: it shows that these differences are embedded in the nature of water rights and in the role that public authorities retain in the rule-making process. The Californian negotiations aim at finding an equilibrium compromise between competitive users in order to avoid litigation. The Californian state sets "credible threats" to force stakeholders to accept trade-offs and to reach a consensus. It uses the negotiation procedure as a non-market process to settle an efficient and sustainable agreement without having to find out about the true preferences of the agents. The French negotiation system focuses more on dialogue, consultation and conflict management. The state uses the negotiations as an information revelation device. Therefore its intervention is more direct and authoritative.
}

\footnotetext{
* Auteur correspondant : thoyer@ensam.inra.fr
} 
Les problématiques liées à la gestion quantitative de l'eau ont évolué rapidement dans la dernière décennie. Cette évolution résulte d'une augmentation de la demande des différents usages, notamment pour l'irrigation, mais aussi d'une prise de conscience plus aiguë des atteintes à l'environnement, et d'une volonté croissante de prise en compte du bien-être des générations futures. On cherche désormais à répondre au problème de disponibilité en eau moins par la mobilisation de nouvelles ressources que par la résorption des inefficacités liées aux techniques et aux choix de gestion.

Dans des contextes variés, on observe la mise en place progressive de cadres législatifs et réglementaires nouveaux, remettant en cause les droits d'eau et les pratiques existants, insistant sur le caractère décentralisé, négocié et collectif des décisions de gestion, et recherchant des mécanismes d'allocation de la ressource plus efficaces. En janvier 1992, la déclaration de Dublin, qui clôture la Conférence internationale sur l'eau et l'environnement, établit comme principe fondateur que la gestion de l'eau doit être fondée sur une approche participative, impliquant les usagers, les planificateurs et les décideurs, et que les décisions doivent se prendre au niveau pertinent le plus décentralisé. En France, la loi sur l'eau de 1992 reprend ces recommandations en prônant la mise en place de procédures de décision locales, concertées et collectives. Enfin, les textes de la Directive cadre européenne sur l'eau adoptée en octobre 2000, dont l'objectif est de contribuer à " assurer un approvisionnement suffisant en eau de surface et en eau souterraine de bonne qualité pour les besoins d'une utilisation durable, équilibrée et équitable de l'eau », spécifient que les États membres devront «encourager la participation active de toutes les parties concernées à la mise en œuvre de la présente Directive » (Article 14).

Cet article compare la façon dont la France et la Californie répondent à cette nouvelle problématique, en fonction de leur histoire, de leur cadre juridique et législatif, et des modes d'expression des préférences collectives. La comparaison, axée principalement sur la gestion de la concurrence entre le secteur agricole et les autres usagers, est construite sur deux volets : la nature des droits d'eau existants et les processus de délégation par lesquels les décisions de gestion de l'eau sont partiellement transférées aux groupes d'usagers concernés. L'article s'appuie sur la comparaison de deux cas d'étude : le bassin de l'Adour dans le Sud-Ouest de la France et la Central Valley en Californie ${ }^{1}$.

\footnotetext{
${ }^{1}$ Les auteurs remercient Dr L. Simon et Prof. G. Rausser de l'Université de Berkeley, California, pour leur collaboration. Cet article fait partie d'un programme d'études financé par le fonds France-Berkeley et par l'Action scientifique structurante AQUAE (Inra/Cemagref).
}

\section{La gestion des droits d'usage sur l'eau}

La définition juridique du droit d'eau est un problème compliqué auquel le droit latin et le droit anglo-saxon apportent des réponses différentes. On le montrera en comparant les réponses respectives des systèmes français et californien à trois questions : comment sont alloués et gérés les droits d'eau? Comment partager la pénurie? Comment introduire de nouveaux droits pour des nouveaux types d'usage? Notre analyse se fondera le plus possible sur les pratiques en vigueur, et non sur une approche juridique formelle.

\section{Allocation et gestion des droits d'eau}

Fondamentalement, aux États-Unis comme en France, les droits d'eau sont des droits d'usage et non des droits de propriété. Il existe des différences fondamentales entre le droit des eaux closes et souterraines et le droit des eaux courantes.

Les eaux closes et souterraines sont traitées dans le régime traditionnel du droit riverain, assez semblable en France et en Californie. Du fait des compétitions sur la nappe ou des interactions entre nappe et cours d'eau, ce droit présente des limites et est donc soumis, en France comme en Californie, à une double disposition légale de déclaration de tout nouvel aménagement et prélèvement, et de garantie de non-gaspillage. En revanche, les eaux courantes ont donné lieu à des traductions formellement assez différentes en Californie et en France.

\section{En Californie}

Le droit des eaux superficielles est régi par deux doctrines fondamentales (Wolfe, 1996; Reisner et Bates, 1990) : celle du droit riverain et celle de la première appropriation. Les droits riverains s'appliquent aux propriétaires fonciers localisés le long des rivières. Ceux-ci sont autorisés à prélever un volume d'eau "raisonnable » tant que cela n'interfère pas avec l'usage « raisonnable » des autres riverains, la notion d'usage raisonnable restant assez floue. Leur droit est théoriquement inaliénable (appropriative water rights).

Mais l'eau est aussi distribuée loin des cours d'eau naturels par l'intermédiaire d'aqueducs et de canaux. Pour les non-riverains, le droit suit la doctrine de la première appropriation : le droit d'usage appartient à celui qui a utilisé l'eau le premier, conformément à l'esprit de conquête qui animait les pionniers. Le droit d'eau confère la possibilité de prélever une quantité d'eau spécifiée pour un usage donné, jugé utile pour la collectivité. La notion de beneficial use range par ordre de priorité les usages domestiques puis agricoles et industriels. Les droits d'eau sont détenus par des usagers (qui sont 
des particuliers ou des associations d'usagers, les plus répandues étant les water districts, équivalents de communautés d'irriguants ou de collectivités urbaines) ou par des sociétés d'aménagement qui ont construit des infrastructures d'acheminement et de distribution d'eau, et qui offrent ensuite des contrats d'eau aux usagers.

Le droit californien distingue les personnes ou les institutions détenant des droits « seniors » établis avant décembre 1914 et celles détenant des droits «juniors » dont l'attribution est, depuis cette date, soumise à la réglementation d'une agence publique, le State Water Resources Control Board (SWRCB). Cette commission de régulation, et à travers elle l'État de Californie, gère et attribue tout nouveau droit : elle enregistre les demandes des nouveaux usagers, vérifie que le besoin est réel et l'usage conforme à l'intérêt public, puis notifie la demande publiquement. Les objections à cette demande sont possibles pendant une période définie, à l'issue de laquelle le SWRCB auditionne les parties en présence, et prend sa décision finale. Les droits acquis antérieurement ne sont pas remis en question et demeurent prioritaires par rapport aux droits nouvellement alloués, qui ne peuvent donc s'exercer que sur des quantités considérées comme excédentaires par rapport aux besoins des ayants droit existants. Le SWRCB peut ainsi déterminer l'ensemble des droits existant sur un cours d'eau donné et déclarer qu'une rivière est totalement appropriée, interdisant ainsi l'attribution de nouveaux droits. Le SWRCB a aussi le pouvoir de confisquer, sans compensation, les droits d'eau lorsqu'ils n'ont pas été utilisés pendant cinq années consécutives. En revanche, lorsque l'État californien ou l'État fédéral exproprient les détenteurs de leurs droits d'eau pour des raisons d'utilité publique, ces derniers sont en droit d'exiger des compensations financières.

Il existe par ailleurs une procédure judiciaire d'adjudication des droits, lorsque des usagers contestent auprès d'un tribunal le bien-fondé des droits existants et réclament une nouvelle allocation. Cette procédure est lente et les conclusions auxquelles elle aboutit sont toujours susceptibles d'être remises en cause par des procédures d'appel ou par d'autres tribunaux dans d'autres juridictions.

L'État californien est donc supposé avoir un pouvoir discrétionnaire sur le statut des droits juniors qui sont donc de fait des droits d'usufruit plutôt que des droits de propriété. Cependant, tout différend sur les droits d'eau pouvant aussi être examiné et traité par les tribunaux, le pouvoir de l'État en termes de ré-allocation des droits reste relativement limité par la menace de procédures judiciaires.

\section{En France}

En France, les eaux courantes navigables et flottables sont propriété publique (domaniale), tandis que les autres eaux courantes relèvent d'une gestion en biens communs, ou res communis (Barraqué, 1995). Les riverains bénéficient d'un accès privilégié à la ressource, ce qui leur confère un droit d'usufruit. En revanche, les propriétaires privés individuels ou les collectivités ayant construit des infrastructures de prélèvement d'eau (canaux d'irrigation gravitaires ou moulins, par exemple) détiennent des droits d'eau spécifiques, généralement consacrés par un titre officiel et exprimés en débit maximum de prélèvement (Le Moal, 1993).

L'accroissement des pressions sur la ressource et la fréquence accrue de conflits entre usagers ont rendu nécessaire une certaine uniformisation du droit, recherchée par la loi sur l'eau de 1992 (Gazzaniga, 1993). Les usages de l'eau sont de plus en plus réglementés, notamment par la généralisation du système d'autorisation de prélèvements et de rejets. Les autorisations de prélèvement sont délivrées annuellement par la Mission inter-services de $l^{\prime} e^{2}{ }^{2}$ (MISE), pour un débit et un usage donnés. En principe, la MISE ne doit délivrer d'autorisation nouvelle de prélèvement que si la ressource est suffisante pour satisfaire l'ensemble des besoins y compris la préservation du milieu naturel. Dans la pratique, ce principe a souvent été mal appliqué et les autorisations de prélèvements, accordées trop facilement, n'ont pas permis un contrôle adéquat des usages. De même, bien qu'elles soient délivrées pour l'année, il est rare de les voir remises en cause d'une année sur l'autre. Il en résulte un principe d'antériorité tacite et une nécessaire gestion de la file d'attente, les nouveaux volumes disponibles étant souvent attribués par ordre de sollicitation.

\section{Partage de la pénurie : priorité ou proportionnalité}

Les systèmes d'antériorité en Californie et de droits d'usage administrés en France impliquent deux procédures différentes de répartition des pénuries d'eau entre les usagers et donc des partages de risque différents.

En Californie, les détenteurs de droits seniors, prioritaires, peuvent utiliser la totalité du volume auquel ils ont droit avant que les juniors ne puissent accéder à l'eau. Les seniors bénéficient ainsi d'une relative sécurité d'accès à l'eau, contrairement aux juniors qui supportent toutes les restrictions en cas de sécheresse. Historiquement, les premiers utilisateurs de l'eau, aujourd'hui détenteurs d'eau seniors, sont des éleveurs qui irriguent des pâtures et de la luzerne, alors que les usages domestiques des grands centres urbains du Sud de la Californie (Los Angeles, San Jose), et les usages agricoles intensifs (arboriculture fruitière, maraîchage), qui se sont développés plus récemment, ne bénéficient que de droits juniors. Dans ces

\footnotetext{
${ }^{2}$ La MISE rassemble, à l'échelle d'un département, tous les services déconcentrés de l'État touchant aux questions de gestion de l'eau.
} 
conditions et en l'absence de mécanisme de ré-allocation des droits, l'application du principe d'antériorité peut entraîner des restrictions pour les consommateurs d'eau potable et fait obstacle au développement de l'agriculture intensive.

En France, une fois les usages prioritaires satisfaits ${ }^{3}$, la pénurie est ensuite répartie, le plus souvent de manière proportionnelle, entre les autres usagers, principalement l'agriculture et l'industrie, par un système de restrictions temporaires de prélèvements prononcées par le préfet de manière ad hoc.

Le droit californien privilégie donc les usagers les plus anciens, tandis que le système français répartit les risques de manière ad hoc entre les usagers, tout en les limitant pour les consommateurs d'eau potable et l'environnement. Cette différence n'est pas sans conséquence sur les possibilités de réforme et sur la participation des usagers au processus de négociation.

\section{Légitimation de nouveaux usages et création de nouveaux droits pour l'environnement}

La dernière décennie a consacré l'apparition de nouveaux usages de l'eau, en particulier les usages non consommateurs comme les loisirs et la protection des milieux naturels. Reconnus comme légitimes, ces nouveaux usages se voient attribuer des droits d'eau, selon des procédures nouvelles et peu stabilisées.

Aux États-Unis, deux lois fédérales légitiment officiellement l'introduction de nouveaux droits en faveur de la protection du milieu : il s'agit de la loi sur la lutte contre la pollution de l'eau (Clean Water Act, initialement publiée en 1972) et celle sur les espèces menacées (Endangered Species Act en 1973) qui spécifient que les objectifs d'amélioration de qualité de l'eau et de préservation des espèces sont prioritaires sur les activités humaines. Elles conduisent à définir, sur des critères scientifiques, des standards de pollution et des débits minimums d'étiage à respecter dans les rivières, équivalents d'un quota garanti pour l'environnement ou de la création de in stream rights.

En France, le système législatif a aussi reconnu officiellement les milieux naturels comme usagers à part entière. La loi Pêche a défini des débits minimums pour la protection des milieux piscicoles dès 1964. La loi sur l'eau de 1992 a été plus loin en consacrant la ressource eau comme patrimoine commun de la nation. Du point de vue du droit, cette notion ${ }^{4}$ excède ainsi « la simple res communis qui n'appartenant à personne était susceptible

\footnotetext{
${ }^{3}$ La loi pêche de 1984, puis la loi sur l'eau de 1992, donnent la priorité aux usages environnementaux et aux consommateurs d'eau potable par la définition de débits minimums.

4 Elle reste cependant ambiguë car elle préserve, du moins dans les textes, les droits d'usage acquis tout en invoquant la responsabilité commune et partagée : le $1^{\text {er }}$ article de la loi de
}

d'être gaspillée » (Sironneau, 1994). En effet, elle suppose la transmission aux générations futures d'une ressource en eau préservée en quantité et en qualité, ouvrant la voie à la notion de gestion durable de l'eau. Comme aux États-Unis, elle se traduit concrètement par la définition de débits minimums d'étiage.

Ces lois ne fournissent pas de règles sur la façon dont doit être répartie entre les autres détenteurs de droits la réduction des prélèvements disponibles. De fait, deux interprétations sont possibles.

La mise en place de débits minimums d'étiage dans les rivières peut être interprétée comme une confiscation aux dépens des droits d'usage existants, même si, dans de nombreuses situations, le financement public d'ouvrages de régulation, assurant une plus grande régularité de l'approvisionnement en eau, représente une forme de compensation à la réduction des droits d'usage. Dans ce premier cas, cette « confiscation » ouvre la porte à des négociations entre les parties prenantes qui selon le contexte peuvent porter sur les compensations éventuelles que les nouveaux usagers devraient verser aux parties lésées ${ }^{5}$; sur le montant des compensations payées par l'État; ou, plus spécifiquement lorsque l'État envisage l' «expropriation », sur une remise à plat des droits existants (Miceli et Segerson, 1995).

Mais l'obligation de respecter des débits minimums peut aussi être interprétée comme une pénurie supplémentaire, qui doit être gérée et partagée conformément au droit de l'eau existant. En Californie, la règle d'antériorité reportera cette pénurie sur les détenteurs de droits juniors dont l'accès à l'eau sera réduit du montant accordé à l'environnement; les droits seniors quant à eux ne seraient affectés qu'en cas de sécheresse très sévère. En France, cette pénurie artificielle sera gérée de façon administrative et proportionnellement aux usages établis.

Dans les deux cas, la création des nouveaux droits est porteuse de conflits politiquement difficiles à gérer, car elle a des conséquences sur le partage du risque entre les acteurs économiques et sur la valeur marginale de la ressource. Les solutions autoritaires et centralisées se heurtent souvent à une résistance forte de la part des parties concernées.

1992 rappelle que l'usage de l'eau appartient à tous «dans le cadre des lois et règlements ainsi que des droits antérieurement établis ».

${ }^{5}$ Dans les négociations sur la redistribution des droits d'eau en amont de la rivière San Joaquin en Californie, il a été proposé que les groupes environnementalistes paient une "compensation » aux détenteurs de droits seniors afin qu'ils prélèvent moins d'eau durant les étiages, ce qui peut s'interpréter comme l'instauration d'un marché de droits. 


\section{L'adoption d'une procédure de décision décentralisée et négociée}

L'originalité des démarches de réforme des systèmes de gestion de l'eau en France et aux États-Unis réside dans l'adoption d'un mécanisme de prise de décision décentralisé et négocié entre les parties prenantes. En théorie, les avantages attendus d'une gestion décentralisée des ressources naturelles reposent sur trois éléments : l'information, la responsabilisation, la légitimité. En adoptant une échelle de gestion locale, plus proche des réalités du terrain, la décentralisation permet de décider à partir d'une base d'information plus précise sur la ressource, ses disponibilités, ses aléas, et sur les acteurs, leurs préférences, leurs contraintes (Caillaud et al., 1996). Elle introduit donc plus de souplesse dans le choix des instruments de gestion et une meilleure adaptation aux conditions locales. Les principes de subsidiarité et d'action collective qui sont généralement associés à la décentralisation sont garants d'une gestion plus autonome et mieux coordonnée, dans laquelle les acteurs se sentent impliqués et responsabilisés par leur participation au processus de décision et de mise en œuvre. Enfin, la recherche d'un consensus entre les acteurs locaux par le dialogue et la consultation favorise les processus d'apprentissage collectif et d'information mutuelle : elle permet de renforcer la légitimité des décisions prises collectivement et donc de les rendre socialement plus acceptables.

Cependant, les processus politiques et les contextes économiques qui ont conduit à l'adoption d'une procédure de décision décentralisée sont très différents en Californie et en France et ont donc favorisé des structures de négociation qui ne reposent pas sur la même philosophie.

Aux États-Unis, où a toujours été affirmée de manière forte la primauté de la propriété privée, c'est la montée en puissance des revendications écologistes qui a rendu nécessaire la réintroduction des principes communautaires dans la gestion de l'eau. L'État californien s'est montré très réticent à engager les réformes nécessaires pour respecter le Clean Water Act et le Endangered Species Act car elles supposaient d'imposer des restrictions importantes aux usagers actuels. Bien que l'Agence de protection de l'environnement (EPA, qui dépend du ministère fédéral de l'Environnement) soit formellement habilitée à faire respecter la loi en décrétant de manière autoritaire une ré-allocation des droits d'eau, le gouvernement fédéral a préféré favoriser l'ouverture de négociations entre les parties prenantes. Les raisons d'un tel choix sont liées principalement à la structure politique (qui exacerbe les tensions entre les choix des États et les contraintes imposées au niveau fédéral) et à l'organisation du pouvoir judiciaire américain : une révision des droits d'eau peut être interprétée comme une entorse aux principes de la propriété privée et des libertés individuelles, et peut donner lieu à un jugement jurisprudentiel en faveur des citoyens "lésés », contre la décision fédérale. Les risques d'une contestation juridique réduisent donc les marges de manœuvre du gouvernement fédéral, qui préfère alors s'appuyer sur des procédures négociées et des solutions contractualisées. Il faut souligner d'ailleurs qu'une loi votée par le Congrès en 1990, le Negotiated Rulemaking Act, recommande aux agences publiques de privilégier les procédures de concertation et de négociation avec les parties prenantes lors de l'élaboration de nouvelles règles ou normes. On observe ainsi en Californie la mise en place progressive, à partir du début des années 1990, d'un processus informel de négociation (the three way water agreement process) entre agriculteurs, consommateurs urbains et groupes de défense de l'environnement (Adams et al., 1996).

En France, la démarche de négociation des règles de gestion de l'eau s'inscrit dans la lignée des lois de décentralisation de 1982-83 puisqu'elle remet aux collectivités territoriales la responsabilité d'élaborer la gestion locale. La loi sur l'eau de 1992, par son souci d'associer dans la concertation, non seulement les collectivités territoriales et les représentants de l'État, mais aussi les usagers, pousse plus loin la logique de la subsidiarité et de la décentralisation. Elle prévoit deux étapes dans le processus de réforme. La première étape établit les grandes lignes de planification de la gestion de l'eau dans chacun des six grands bassins hydrographiques français : ce sont les Schémas directeurs d'aménagement et de gestion des eaux (SDAGE). La deuxième étape prévoit la mise en place de négociations entre toutes les parties prenantes sur les outils de gestion à établir à l'échelle locale pour respecter les contraintes des SDAGE et pour mieux entretenir les ressources locales (Schéma d'aménagement et de gestion des eaux (SAGE)). Cette deuxième étape, facultative bien que fortement encouragée, doit être initiée par les acteurs locaux ${ }^{6}$.

Aux États-Unis comme en France, les procédures de décision négociée restent relativement nouvelles. Même si on constate qu'elles se multiplient dans le domaine de l'environnement et des politiques publiques territoriales,

\footnotetext{
${ }^{6}$ Les démarches de concertation avaient déjà été partiellement expérimentées dans le cadre des contrats de rivière institués par la circulaire du ministre de l'Environnement du 5 février 1981. Il s'agit d'un accord technique et financier entre un ou plusieurs maîtres d'ouvrages locaux couvrant l'ensemble d'un bassin versant, l'État, la Région, le Département, l'Agence de l'eau et les usagers, pour améliorer la gestion environnementale d'une rivière. Le SAGE n'a pas remplacé le contrat de rivière. Il s'adresse aux mêmes acteurs, s'inspire des mêmes principes et poursuit un même objectif opérationnel : développer un mode de gestion équilibré à l'échelle d'un bassin versant. Le SAGE revêt toutefois une dimension supplémentaire : sanctionnées par un arrêté préfectoral, ses orientations ont une portée réglementaire en devenant le cadre de planification de la politique locale de l'eau.
} 
les pouvoirs publics restent démunis pour mettre en œuvre de telles politiques. L'une des principales difficultés concerne la structure de la négociation : comment décider quels intérêts doivent être représentés et avec quel poids? Les risques d'une sur-représentation des intérêts économiques et d'une sous-représentation des intérêts non directement économiques (consommateurs urbains, environnement) peuvent conduire à des décisions biaisées qui reflètent plus l'agrégation des préférences pondérée par les poids politiques des acteurs participant effectivement à la négociation, que la recherche d'un optimum social de long terme. En effet, si, selon la théorie ${ }^{7}$, un cadre de négociation sans contrainte doit conduire, par analogie à un marché de concurrence pure et parfaite, à un optimum pour les intérêts économiques privés, en revanche, il peut conduire à une sous-production des externalités positives et à une surproduction des externalités négatives, dont respectivement les bénéficiaires et les victimes sont en général mal identifiés et peu organisés (effets diffus, effets à long terme, effets non directement observables). En dehors des travaux fondateurs de Coase, la littérature économique n'offre que des réponses limitées (Kazmierczak et Hughes, 1996) à la question de la procédure optimale de négociation. Les recherches de sociologie en environnement sont plus riches et se sont intéressées aux conditions à réunir pour favoriser les prises de décision fondées sur le consensus, en explorant plus particulièrement le rôle des médiateurs (Pellow, 1999; Beuret et Trehet, 2001).

Les systèmes de droits d'usage en France et en Californie, et l'organisation institutionnelle administrant ces droits, sont déterminants pour comprendre les différences de procédures adoptées. L'étude de deux bassins particuliers, la Central Valley en Californie et le bassin de l'Adour en France, permettra d'identifier ces différences.

\section{Les processus de négociation dans la Central Valley en Californie et dans l'Adour en France}

Cette question de la structuration de la négociation sera abordée à travers l'étude : (i) des enjeux des réformes; (ii) des étapes de la négociation; (iii) des incitations à négocier et du poids respectif des parties négociantes.

\footnotetext{
${ }^{7}$ Lindahl démontre que les négociations entre consommateurs contribuables permettent d'établir des règles fiscales pour le financement des biens collectifs qui se confondent avec l'optimum parétien. Samuelson étend cette théorie à un modèle d'équilibre général.
}

Les enjeux des réformes : une concurrence accrue entre les usages

La Central Valley en Californie, d'une longueur de $700 \mathrm{~km}$, est un vaste ensemble hydrographique formé de la San Joaquin River et de la Sacramento River, qui coulent au pied de la Sierra Nevada et se rejoignent pour former le delta avant de se jeter dans la baie de San Francisco. Ce bassin versant couvre $37 \%$ de la surface de l'État californien. Malgré les nombreux aménagements de barrages réservoirs, financés par l'État fédéral (le Central Valley Project qui est géré par le Reclamation Bureau USBR) ou par l'État de Californie (le State Water Project géré par le Department of Water Resources), les ressources en eau demeurent largement insuffisantes en année normale pour satisfaire tous les usages, notamment les besoins d'irrigation qui représentent $50 \%$ des usages mais aussi, et de plus en plus, les besoins en eau potable d'une population en forte croissance dans les grands centres urbains $\mathrm{du}$ bassin (San Francisco, Sacramento) et hors bassin ${ }^{8}$ (dans la région de Los Angeles).

Du fait de ces aménagements et de l'augmentation de la consommation, la zone du Bay-Delta, autrefois célèbre pour ses zones humides et sa biodiversité, a connu de profondes altérations écologiques. Les prélèvements excessifs sur les fleuves Sacramento et San Joaquin provoquent des intrusions d'eau salée de la baie de San Francisco dans le delta, ce qui dégrade la qualité des eaux, menace l'équilibre écologique des marais et la survie des poissons. Ces phénomènes ont été accentués par les sécheresses sévères de 1976-77 et de 1987-91. Deux espèces piscicoles du delta sont désormais inscrites sur la liste des espèces menacées. En conséquence, dès le milieu des années 1980, et en conformité avec les lois fédérales, le SWRCB a cherché à élaborer un plan de gestion de l'eau destiné à assurer un quota d'eau minimum pour la préservation du milieu naturel, mais impliquant des réductions substantielles des quantités prélevées par les zones urbaines et agricoles. Schématiquement, deux alternatives extrêmes ont été initialement envisagées pour répartir ces réductions :

- soit le renforcement des droits d'eau actuels associé au développement des marchés de l'eau de façon à faciliter le transfert vers les usages les plus valorisants (c'est l'option de sécurisation des droits d'eau-secure water rights alternative);

- soit une répartition des restrictions favorisant les usagers urbains du Sud de la Californie et les productions ayant une valeur marginale de l'eau élevée (c'est l'option dite «juste » - fair alternative) mais ayant l'inconvénient de remettre en question le système de droits existant.

\footnotetext{
$825 \%$ du volume moyen entrant annuellement dans le bassin versant sont exportés hors bassin.
} 
La première solution ayant été rejetée par le gouverneur de Californie de l'époque, qui soutenait les intérêts des entreprises du secteur agro-alimentaire et des districts municipaux d'eau du Sud de la Californie, c'est donc vers la seconde alternative que se sont tournés les pouvoirs publics en mettant en place les conditions d'une solution négociée.

Le bassin versant amont de l'Adour, qui représente un espace plus limité de l'ordre de $50 \mathrm{~km}$ de rivière, connaît lui aussi un déficit de la ressource important ${ }^{9}$. Contrairement à la Californie, le déséquilibre peut ici être attribué presque exclusivement à la croissance forte de la demande en eau pour l'irrigation qui représente l'essentiel des prélèvements ( $65 \%$ en moyenne sur l'année mais jusqu'à $80 \%$ en période d'étiage).

Malgré la mise en place des autorisations de prélèvements depuis la loi sur l'eau de 1992, la gestion administrative des usages n'a pas permis de maintenir la qualité de l'eau des rivières. Dans ce contexte de concurrence croissante entre les usages (usage agricole, eau potable, usages récréatifs), accentué par les fortes sécheresses des deux dernières décennies, l'ensemble des acteurs de la gestion de l'eau décide en 1996 de : (i) mettre en place des outils de connaissance de la ressource en eau (tableau de bord Adour); (ii) mobiliser de nouvelles ressources par la construction de plusieurs barrages ; (iii) d'établir de façon négociée des règles de répartition de la ressource entre les usages en année normale et en année sèche (plan de gestion des étiages). L'enjeu de la négociation que nous étudions ici porte sur la nature de ces règles de gestion, et sur la répartition entre les différentes parties prenantes des coûts de ce nouveau mode de gestion.

\section{Les étapes de la négociation}

Dans le cas californien, comme dans l'Adour, la négociation a eu lieu en deux étapes qui se résument ainsi : une première phase de négociation fixe les niveaux de débit minimum dans les rivières, une décision collective sur le quota d'eau accordé à l'environnement aux dépens des autres usages; la deuxième phase est consacrée à la recherche d'un compromis sur la répartition des contraintes imposées par cette politique de débit minimum.

\section{En Californie}

Constatant l'incapacité de l'État californien à mettre en place les réformes nécessaires, le gouvernement fédéral fait le premier pas et choisit d'imposer les réformes

\footnotetext{
${ }_{9}$ Environ $60 \mathrm{Mm}^{3}$ par an (Institution interdépartementale pour l'aménagement hydraulique du bassin de l'Adour, 1994).
}

dans le domaine qu'il contrôle. Il édicte en 1992 le Central Valley Project Improvement Act ${ }^{10}$ (CVPIA) qui impose des modifications dans la gestion du Central Valley Project afin d'en réduire les impacts environnementaux : il prévoit notamment un volume d'eau de près de 1000 millions de $\mathrm{m}^{3}\left(\mathrm{Mm}^{3}\right)$ annuels dédié à l'environnement. Il restreint la mise en place de nouveaux contrats de fourniture d'eau et encourage la mise en place de marchés de l'eau entre agriculteurs et consommateurs urbains. Mais les contraintes imposées par le niveau fédéral ne concernent que les aménagements qu'il gère, soit à peine $20 \%$ de l'offre en eau californienne.

Il faut attendre décembre 1994 pour aboutir à un accord général touchant l'intégralité de la zone du BayDelta (The Bay-Delta Accord) signé pour une période de trois ans (prolongée ensuite jusqu'à fin 1998). Cet accord est le premier succès des négociations tripartites CalFed réunissant depuis le début des années 1990 sous la double houlette du pouvoir fédéral et de l'État californien, les représentants des consommateurs urbains, les représentants d'irrigants et les groupes de défense de l'environnement. Il définit des objectifs de qualité de l'eau qui conduisent à restreindre les prélèvements de $1350 \mathrm{Mm}^{3}$ les années sèches et à imposer des volumes maximum prélevables mensuels. Il est décidé de reconduire la structure CalFed qui donne un contenu plus formel à la négociation tripartite avec pour mission de contribuer à la recherche de solutions de long terme aux problèmes d'environnement, de fiabilité de l'offre et de qualité de l'eau.

La seconde phase de négociation n'a véritablement commencé qu'en 1998 après la publication de plusieurs rapports d'expertise commandités par CalFed, chargés de faire des propositions sur les manières de répartir les restrictions. C'est la base des négociations, animées par le SWRCB sous la forme de water rights hearings. Toutes les parties prenantes (environ 200) sont invitées à ces discussions très structurées, menées sur la base d'un calendrier précisé à l'avance visant à favoriser les accords partiels. Ainsi, une proposition de partage des droits et de modalité de règlement des conflits a été déposée en 1998 pour la gestion des eaux sur le fleuve San Joaquin (San Joaquin River Agreement). Cette offre d'accord partiel a émergé spontanément, sur l'initiative des détenteurs de droits seniors, inquiets de la menace qui pesait sur eux d'une perte de leurs droits. Les seniors proposent de vendre de $\mathrm{l}^{\prime} \mathrm{eau}^{11}$ à l'environnement pour que les standards de qualité et de quantité puissent être atteints. En contrepartie, outre une compensation financière qui pourrait être versée par le fonds de restauration (géré par le département

\footnotetext{
10 Aussi connu comme le Miller-Bradley bill.

11 L'accord portait sur la mise à disposition par le groupement des détenteurs de droits seniors de la San Joaquin River (SJRGA) de $134 \mathrm{Mm}^{3}$ à 0.05 US\$ le $\mathrm{m}^{3}$, pour satisfaire les débits minimums.
} 
californien de l'environnement), ils exigent l'assurance que la pérennité de leurs droits soit assurée et ne soit plus un enjeu négociable. D'autres accords partiels sont ensuite proposés. La dernière phase de négociation, qui a pris fin en décembre 1999 avec la décision 1641 du SWRCB, a entre autres examiné les conséquences de ces accords pour les autres parties. La décision finale entérine les solutions proposées dans ces accords partiels pour le respect des objectifs de débits définis dans le plan de contrôle de la qualité des eaux et modifie les droits d'eau en conséquence ainsi que la gestion hydraulique du système global.

\section{En France}

Le SDAGE du bassin Adour-Garonne a été signé en 1996, dans les délais imposés par la loi de 1992. C'est un outil de planification qui définit, entre autres, les débits objectifs d'étiage (DOE) et les débits de crise (DCR) en différents points nodaux du bassin ${ }^{12}$.

La deuxième phase de négociation a été entamée avec la mise en place début 1998 de la négociation sur le Plan de gestion des étiages (PGE) de l'Adour recherchant un compromis sur les règles de gestion qui permettent de respecter les débits minimums fixés par le SDAGE. Ce plan a finalement été signé en février 1999 par l'ensemble des parties prenantes. Il comporte un état des lieux des ressources et des besoins en eau, établissant l'importance du déficit. Les solutions proposées pour résorber ce déficit comportent des mesures d'économies d'eau au niveau des prélèvements et de la gestion des ouvrages de stockage et de transfert d'eau $\left(15 \mathrm{Mm}^{3}\right)$, l'utilisation pour le soutien d'étiage $\left(4 \mathrm{Mm}^{3}\right)$ d'une retenue existante, dont la vocation actuelle est la production d'énergie, et enfin la création de deux nouveaux barrages réservoirs $\left(25 \mathrm{Mm}^{3}\right)$. Un échéancier précis de la mise en place de ces différentes mesures a été établi.

Les DOE définis dans le SDAGE ne sont pas remis en cause mais une réévaluation progressive des DCR est prévue suivant la mise en place des nouvelles capacités de stockage. Ces valeurs devront être intégrées dans la future révision du SDAGE, leur conférant une portée juridique. Les règles de gestion retenues portent sur les volumes globaux d'eau pouvant être prélevés huit années sur dix, compatibles avec les DOE, et leur répartition entre les différents usages. Des pénalités financières sont prévues en cas de dépassement des

\footnotetext{
${ }^{12}$ Le débit objectif d'étiage est la valeur «au-dessus de laquelle sont assurés la coexistence normale de tous les usages et le bon fonctionnement du système aquatique » (Comité de bassin Adour-Garonne, 1996). Le débit de crise correspond à la valeur " au-dessous de laquelle sont mises en péril l'alimentation en eau potable et la survie des espèces présentes dans le milieu ». En deçà de cette valeur, des restrictions de prélèvements sont prononcées par les préfets.
}

volumes maximums prélevables. Les usagers, notamment agricoles, s'engagent, d'une part, à mettre en place un programme d'information et de formation sur les économies d'eau, d'autre part, à contribuer financièrement aux coûts de fonctionnement, entretien et maintenance des ouvrages construits (coûts estimés à $10 \mathrm{MF}$ par an). Le contenu des plans de crise est précisé. Les financements publics sont conditionnés, pour la construction des ouvrages de stockage, à l'élaboration du PGE et, pour les équipements d'hydraulique agricole, au respect des mesures qu'il contient. Une commission de gestion rassemblant les acteurs et usagers de l'eau ayant participé à son élaboration devra assurer le suivi de la mise en œuvre du PGE.

Il est intéressant de noter la préférence accordée, en Californie comme en France, aux intérêts écologiques dont les «droits d'eau » sont discutés avant d'aborder la question du partage des droits d'eau pour les autres usages. Dans les deux cas, l'attribution d'un quota garanti à l'environnement révèle l'inquiétude des autorités devant le poids minoritaire des intérêts environnementaux, qui les conduit à imposer des normes minimales de protection de l'environnement. Mais ces normes, déterminées de manière exogène sur des critères d'experts, sont souvent jugées inacceptables par certaines parties prenantes. Il est reconnu que le fait de soustraire à la négociation l'un de ses paramètres s'oppose à l'objectif de consensus et en fait paradoxalement un enjeu crucial de la négociation ${ }^{13}$ (Caldart et Ashford, 1998). De fait, les négociations n'ont pu démarrer dans les deux cas que lorsque l'État s'est engagé à ce qui a pu être perçu comme des concessions ${ }^{14}$ vis-à-vis des parties prenantes, témoignant de la bonne volonté des autorités publiques à participer à l'effort collectif.

Une autre étape déterminante est celle de 1 " « état des lieux » de la ressource, l'accord obtenu sur le diagnostic de la situation et sur le volume de la ressource apparaissant comme un préalable aux discussions. Si un diagnostic exhaustif et accepté par tous n'existe pas, les acteurs ne semblent prêts à s'engager que s'ils reconnaissent comme acceptable un diagnostic commun.

\section{Les incitations à participer et les règles de participation}

La coopération entre des usagers ayant des intérêts divergents est rarement spontanée; elle résulte de

\footnotetext{
13 Ceci est illustré notamment dans l'Adour, par la renégociation à la baisse des débits de crise après la signature du SDAGE (Faÿsse, 1998).

14 Le Central Valley Project Improvement Act qui s'engage à moderniser le réseau, à limiter l'entrée de nouveaux usagers et à favoriser institutionnellement et financièrement des solutions marchandes entre usagers; l'engagement de l'État français a participer aux frais d'investissement de nouveaux barrages.
} 
mécanismes les incitant à coopérer et à participer qui reposent sur des principes très différents en Californie et en France.

En Californie, les parties prenantes sont incitées à participer du fait de la menace crédible des autorités fédérales qui sont en droit d'imposer pour raison de non-conformité aux lois environnementales, des mesures répressives, en particulier une révision autoritaire des droits d'eau selon des règles qu'elles ne révèlent pas. Sous cette menace, tous les groupes d'usagers ont intérêt à jouer le jeu d'une négociation et à trouver un compromis : les détenteurs de droits juniors parce que, si le principe d'antériorité est maintenu ou renforcé, ils devront supporter entièrement le poids des restrictions d'eau ; les détenteurs de droits seniors parce que, faisant en général un usage peu intensif de l'eau, leurs droits pourraient être partiellement remis en cause au profit des juniors.

La table des négociations californienne est ouverte à tous les groupes qui font une déclaration d'intention de participation. Elle fonctionne sous forme de public hearings qui donnent l'occasion à toutes les parties prenantes de défendre publiquement leurs positions. Sa composition n'est donc pas définie au préalable mais émerge comme le résultat d'une auto-sélection des intérêts les plus motivés.

Les participants à la négociation californienne, à la première comme à la seconde étape, sont généralement l'agence de l'environnement californienne (département de l'eau, et département de la chasse et de la pêche); les services chargés de la gestion des parcs nationaux (en particulier le service de protection de la faune sauvage et celui de l'assainissement) ; les communautés d'irrigants (water districts et mutual companies); les grands centres urbains qui peuvent avoir des droits relativement seniors comme San Francisco et les municipalités « exportatrices » qui exportent de l'eau hors du bassin versant (Los Angeles); des associations de protection de la nature; des groupes industriels, en particulier de production d'hydroélectricité ; les institutions gestionnaires des grands projets, etc. Les public hearings peuvent donc rassembler jusqu'à 200 parties prenantes dont certaines sont des émanations directes des autorités publiques californiennes ou fédérales lorsqu'elles ont des intérêts financiers liés à l'eau.

En France, au contraire, le cadre des négociations est fixé par la loi. Cependant, alors que les SDAGE ont été établis avec un calendrier précis pour les six grands bassins français à l'initiative du préfet coordonnateur de bassin et par les Comités de bassin ${ }^{15}$, les négociations

\footnotetext{
${ }^{15}$ La composition des Comités de bassin a été définie par la loi sur l'eau de 1964 avec trois collèges comptant chacun pour un tiers des voix : les représentants de l'État, les collectivités locales et les représentants d'usagers. Cette répartition a été modifiée en 1984, suite aux lois de décentralisation. Aujourd'hui, les représentants de l'État sont minoritaires, les élus locaux occupent
}

locales de type SAGE ou Plan de gestion des étiages sont laissées à l'initiative locale et ne sont donc théoriquement engagées que si un nombre suffisant de parties prenantes locales demandent l'ouverture de la négociation. Cependant, à l'instar du cas californien, l'État français joue aussi un rôle de «catalyseur » de la négociation. En effet, la participation financière de l'État et des collectivités locales dans les investissements de structures destinées à améliorer les conditions techniques d'accès à la ressource en eau (barrages, rénovation des périmètres irrigués, etc.) est le plus souvent rendue conditionnelle à la mise en place d'un SAGE, qui est censé garantir la durabilité du système de gestion en conformité avec la loi de 1992. Les autorités publiques exercent donc une incitation à la participation par leur statut d' "offreurs d'eau » potentiels. Dans le cas de l'Adour, la construction de trois nouveaux barrages réservoirs est ainsi conditionnée par la signature officielle du plan de gestion des étiages.

Contrairement au cas californien, la composition des instances de concertation est soigneusement consignée dans la loi. Ainsi, pour l'élaboration des SAGE, la loi exige qu'une Commission locale de l'eau (CLE) soit créée, composée de trois collèges avec des droits de vote fixés : pour moitié des représentants des collectivités territoriales et des établissements publics locaux; pour un quart des représentants de l'État; pour un quart des représentants des usagers, des propriétaires riverains, des organisations professionnelles et des associations concernées. La comparaison de la composition des CLE et des Comités de bassin montre que la loi sur l'eau de 1992 s'inscrit d'abord dans une logique de décentralisation, par le renforcement du poids des collectivités territoriales, plus que dans une logique de délégation aux usagers. D'ailleurs, il est clair que l'État reste le maître d'œuvre de cette nouvelle forme de gouvernance qu'il a initiée en commandant depuis 1985 une série de rapports sur la modernisation des droits d'eau, la valeur de la ressource en eau, et la rénovation des structures de gestion (Sironneau, 1994). Il l'a institutionnalisée par la loi sur l'eau de 1992. Il la contrôle par le renforcement du pouvoir de la police des eaux (Ségur, 1993) et par la participation importante de ses représentants aux instances de décision. Les modalités d'élaboration du PGE sont assez proches de celles du SAGE (initiative locale), elles diffèrent par la composition de la commission d'élaboration qui laisse légèrement plus de place aux usagers de l'eau.

Les déterminants de l'action collective sont donc différents en France et en Californie. L'État français a choisi de promouvoir le processus de décentralisation par la voie législative. La décentralisation permet, en transférant une partie des compétences de l'État aux collectivités

plus du tiers des sièges, usagers et milieux socioprofessionnels forment toujours le groupe le plus important (Nicolazo, 1997). 
locales, de réduire les coûts d'information. L'accent est mis sur le dialogue et la consultation pour une conciliation optimale des usages. L'État utilise donc le processus de concertation afin d'acquérir de l'information sur les préférences réelles des parties prenantes et d'affiner ses décisions. Cela explique qu'il souhaite participer de près à la négociation et qu'il en reste le superviseur ${ }^{16}$.

Cette démarche s'oppose à la négociation californienne, qui est plus clairement celle de la recherche d'un compromis entre des usagers en concurrence et dans laquelle l'État n'intervient que par la fixation d'un point de menace incitant les parties à entrer dans une logique de marchandage et à trouver une solution de partage négociée.

\section{Conclusion}

Pour réformer leur système de gestion de l'eau et mieux concilier les différents usages de l'eau, la Californie comme la France ont choisi d'adopter une démarche décentralisée alliant une procédure de négociation entre usagers à un principe de délégation des décisions des pouvoirs centraux aux pouvoirs locaux. Dans les deux cas, c'est une démarche relativement novatrice - qu'on pourrait presque qualifier d'expérimentale - qui donne lieu à des tâtonnements institutionnels.

Les différences dans les deux cas d'étude sont avant tout liées aux caractéristiques du système de droit et aux initiatives de l'État : le principe d'antériorité californien oriente les enjeux de la négociation autour du partage de la pénurie, alors que le droit d'usage administré français pose la question du partage de la ressource en eau dans son ensemble. Les autorités californiennes ont choisi une voie non interventionniste et envisagent la négociation comme le moyen d'obtenir un accord entre usagers, sans avoir à acquérir une information coûteuse sur les vraies préférences des parties prenantes. L'État français, au contraire, reste très présent dans la procédure de négociation qui s'apparente alors plus à un mécanisme de concertation et de réduction des asymétries d'information entre les autorités publiques et les agents privés. Plus qu'une délégation d'autorité, la procédure apparaît comme une forme de gestion des conflits qui passe par la construction d'une information commune et d'objectifs collectivement acceptés. Alors que l'État agit en Californie comme un point de menace pour inciter les participants à trouver un compromis, il garde en France son double rôle d'arbitre (en particulier par la définition

\footnotetext{
${ }^{16}$ L'État maintient ses prérogatives de décideur en dernier ressort puisque les projets de SAGE et de SDAGE doivent obtenir l'approbation du préfet pour être applicables.
}

des règles de la négociation) et de décideur en dernier ressort.

Les recommandations empiriques pour l'amélioration des procédures insistent surtout sur la qualité du consensus obtenu (en termes d'acceptabilité et de résilience) et sur l'analyse, dans une perspective dynamique, de l'adéquation des solutions retenues avec les objectifs de long terme, tels la préservation de la vie aquatique ou l'amélioration des aménités (Sofaer, 1998 ; Barraqué, 1997). Or, il ressort de cette analyse des deux cas d'étude que l'issue de la négociation dépend fortement du pouvoir de négociation des parties prenantes et de la structure de la négociation. Parmi les besoins de recherche, on voit donc la nécessité de substituer aux outils classiques d'aide à la décision des outils d'aide à la négociation. Ceux-ci permettraient d'explorer l'espace des solutions possibles sous différentes hypothèses concernant la structure de la négociation, les arguments négociés, la distribution du pouvoir de négociation. Ces besoins sont d'autant plus cruciaux que les politiques actuelles tendent à renforcer les procédures de concertation et de négociation dans la gestion des biens environnementaux.

\section{Références}

Adams, G., Rausser, G. and Simon, L., 1996. Modelling multilateral negotiations: an application to California water policy. Journal of Economic Behaviour and Organisation, 30, 97-111.

Barraqué, B., 1995. Les Politiques de l'eau en Europe, Paris, La Découverte.

Barraqué, B., 1997. Subsidiarité et politique de l'eau, in Faure, A (Ed.), Territoires et subsidiarité : l'action publique locale à la lumière d'un principe controversé. Paris, L'Harmattan, 165-201.

Beuret, J.E., Trehet, C., 2001. Pour la gestion concertée de l'espace rural : appuyer des médiations territoriales, Le Courrier de l'environnement de l'INRA, 43, 25-39.

Caillaud, B., Jullien, B. et Picard, P., 1996. Information et organisation des processus de décision publique, Revue d'économie politique, 106, 1, 13-35.

Caldart, C., Ashford, N., 1998. Negotiation as a means of developing and implementing environmental policy, Working paper, MIT.

Comité de bassin Adour-Garonne, 1996. Schéma directeur d'aménagement et de gestion des eaux.

Faÿsse, N., 1998. L'Eau entre État et usager dans le bassin de l'Adour : de la gestion réglementaire à la gestion concertée? Mémoire de DEA, Université Montpellier II, Montpellier.

Gazzaniga, J.-L., 1993. La loi du 3 janvier 1992 : une nouvelle politique de l'eau? Revue de droit rural, 218, 445-448.

Institution interdépartementale pour l'aménagement hydraulique du bassin de l'Adour, 1994. Schéma directeur de gestion des étiages de l'Adour.

Kazmierczak, R.F. and Hughes, D.W., 1996. Reasonable Value and the Role of Negotiation in Agriculture's Use of the Environment. Staff paper 96-09, Bâton Rouge, Lousiana State University Agricultural Center. 
Le Moal, R., 1993. Les droits sur l'eau, Revue de droit rural, 218, 449-465.

Miceli, T.J. and Segerson, K., 1995. Government regulation and compensation for takings : implications for agriculture. American Journal of Agricultural Economics, 77, 1177-1182.

Nicolazo, J.L., 1997. Les Agences de l'eau, Paris, P. Johanet et fils.

Pellow, D., 1999. Negotiation and confrontation : environmental policymaking through consensus, Society and Natural Resources, 12, 189-203.

Reisner, M. and Bates, S., 1990. Overtapped oasis. Reform or revolution for western water, Washington, D.C., Island Press.
Ségur, P., 1993. L'accroissement des pouvoirs du préfet dans le cadre de la loi sur l'eau du 3 janvier 1992, Revue de droit rural, 218, 474-482.

Sironneau, J., 1994. La genèse de la loi sur l'eau, Revue de droit rural, 225, 358-360.

Sofaer, A., 1998. California and Colorado-river water: seize the opportunity, in Sanchez, R., Woled, J., Tilly, D. (Eds), Proceedings of the First Rosenberg International Forum on Water Policy, Water Resources Report 93, University of CaliforniaDavis, 9-23.

Wolfe, M.E., 1996. A Landowner's Guide to Western Water Rights. Boulder, Colorado, Roberts Rinehart Publishers.

Reçu le 6 mai 2003. Accepté le 6 novembre 2003. 